\title{
Characterization and dielectric properties of magnetic nanoparticles (Ferrofluid) conjugated with chemotherapy drug for medical application
}

\author{
R. M .Ahmed*, Maha Fadel ${ }^{* *}$, Magda S.Hanafy ${ }^{*}$, Manar A. Ibrahim* \\ * Physics Department, Faculty of Science, Zagazig University, Egypt \\ **National institute of Laser Enhanced Sciences, Cairo University, Egypt.
}

\begin{abstract}
Basically, magnetic drug delivery system using magnetic nanoparticle carriers targeted by an external magnetic field has been recently a promising choice to avoid problems coupled with usual chemotherapy. An external magnetic field is used to localize the drug loaded carriers at the tumor site and the drug can then be released from the carriers. Consequently, our study is concerned with the ferrofluid $\left(\mathrm{Fe}_{3} \mathrm{O}_{4}\right)$ as magnetic nanoparticles coated with PEG (polyethylene glycol) and conjugated with the doxorubicin as an anti-cancer drug in which the doxorubicin mixed with different four amounts of ferrofluid 700,800,900 and $1100 \mu \mathrm{l}$ respectively. Herein, characterization of the ferrofluid $\left(\mathrm{Fe}_{3} \mathrm{O}_{4}\right)$ were carried out using $x$ - ray. Transmission electron microscope (TEM) technique was used for all the mixed samples to define the shape of conjugation. FT-IR method was used in wave number range from 400 to $4000 \mathrm{~cm}^{-1}$ to characterize the structure of the samples. Moreover, the dielectric properties of the samples, such as dielectric constant, $\varepsilon^{\prime}$, dielectric loss $\varepsilon^{\prime \prime}$ and $A C$ conductivity, for these prepared samples were investigated in the frequency range from $0.5 \mathrm{kHz}$ to $1 \mathrm{MHz}$ at $25 \pm l^{\circ} \mathrm{C}$. Our study has been extended to include measurements of absorption spectrum of the samples in wave length range from 300 to $900 \mathrm{~nm}$. The results showed that the samples were characterized by three peaks at wave numbers 490, 1637 and $3459 \mathrm{~cm}^{-1}$ by using FT-IR method. In addition to that TEM technique explained the shape of conjugation of doxorubicin with ferrofluid. Ferrofluid spread on DOX and caused increasing in the darkening of the film due to increasing of their amounts. Also, the conductivity of the samples changed with increasing the ferrofluid amounts. Both of the dielectric constant, $\varepsilon^{\prime}$ and dielectric loss $\varepsilon^{\prime \prime}$, , showed slight changes. The absorption peaks of the samples increased with increasing the ferrofluid amounts loaded with the doxorubicin and the absorption bandwidth became broader.
\end{abstract}

Keywords: Ferrofluid, Doxorubicin, x-ray, FT-IR, Dielectric properties, AC conductivity

\section{Introduction}

Targeted drug delivery systems have been developed to optimize regenerative techniques. The system is based on a method that delivers a certain amount of a therapeutic agent for a prolonged period of time to a targeted diseased area within the body. This helps maintain the required plasma and tissue drug levels in the body therefore, avoiding any damage to the healthy tissue via the drug.

Application of magnetic nanoparticles (MNPs) ferrofluids are numerous. An essential significance of magnetic nanoparticles also includes its medical applications, for instance in magnetically controlled drug targeting, based on the binding of establishes anticancer drugs with ferrofluids that concentrate the drug in the area of interest (a tumor) by means of magnetic fields. In this kind of treatment, the magnetic fluid or ferrofluid is injected directly into the tumor, where it remains by the help of a permanent magnet directly placed over the external side of the tumor ( Rosensweig R. E, 2002).

Ferrofluids are stable suspensions of colloidal ferromagnetic particles (e.g. magnetite) in suitable, non-magnetic carrier liquids. Typical diameters of the colloidal particles are of the order of $10 \mathrm{~nm}$ and are therefore stable against sedimentation. The colloidal particles are covered with surfactants in order to prevent agglomeration due to attractive Van der Vaals forces.

Magnetic biomaterials must present additional characteristics in comparison with materials used for other applications. In this sense, it appears reasonable the use of iron oxide nanoparticles as magnetic core encapsulated in a protective polymeric shell. For example, polymer molecules like polyethylene glycol (PEG) or dextran are known to reduce the opsonisation phenomenon and to lengthen the duration of circulation of the nanovectors (Harris and Chess, 2003). In addition, the coating can provide various chemical groups to conjugate drugs or targeting ligands (Wang and Thanou, 2010).

Polyethylene glycol (PEG) is known to be an attractive material to reduce opsonization because it is uncharged, hydrophilic, and non-immunogenic ( Shan et al., 2009; Karakoti et al., 2011 ). The presence of PEG chains on the NP surface has been shown to increase both their colloidal stability in aqueous suspension ( Herve et al., 2008) and the blood half-life in vivo ( Lankveld et al 2011). 
Doxorubicin (DOX) is an antineoplastic agent of the anthracycline family frequently used in association with other drugs to treat a large number of cancers, like leukemia, ovarian cancers and particularly last stage breast cancers. The clinical use of DOX is limited by its side effects, the most dangerous being a cumulative dose-dependent cardio-toxicity. To minimize the side effects, DOX can be vectorized, that is associated to drug carriers that will favor its accumulation on the site of action and limit its dispersion in healthy tissues .

For using ferrofluid as magnetic nanoparticles after conjugation with doxorubicin in medical application some properties of the samples must be studied. After conjugation of four different amounts of ferrofluid with doxorubicin respectively we define the suitable sample for using in medical application.

The aim of the present study is to characterize the doxorubicin loaded with different amounts of ferrofluid 700,800,900 and $1100 \mu \mathrm{l}$ respectively by using each of FT-IR method and Transmission electron microscope (TEM) technique. Moreover, the dielectric properties of the mixed samples and their absorption spectra were also studied

\subsection{Materials}

\section{Materials and Experimental Method}

Ferrofluid coated with polyethylene glycol $\left(\mathrm{M}_{\mathrm{w}}=400 \mathrm{~g} \cdot \mathrm{mol}^{-1}\right)$ has been purchased from Material science lab, Physics Department, Faculty of Science, Cairo University, Egypt.On the other hand, Doxorubicin hydrochloride (Adricin ${ }^{\circledR}$ ) manufactured by EIMC united Pharmaceuticals (Badr city, cairo, A.R.E ).Also, N[(2-Hydroxy) peperazine-N-(2- ethanosulphonic acid)] buffer (HEPES) was purchased from Sigma Chemical Co (St. Louis, USA)

\subsection{Preparation of the samples}

A diluted buffer with deionized water has a concentration $0.01 \mathrm{M}$ has been prepared which was used to dilute the doxorubicin and the ferrofluid to have a ratio 1:19 $\mathrm{ml}$ for Dox : buffer and 1:30 $\mathrm{ml}$ for ferrofluid : buffer. One ml of diluted Doxorubicin has been used for all the studied samples as a pristine sample and named sample A. Sample A has been loaded with different amounts of the of diluted ferrofluid as following: 700 $\mu 1$, $800 \mu 1,900 \mu 1$ and $1100 \mu 1$ which named samples: B, C, D and E, respectively. For each time of any measurement, the previous samples were prepared to be fresh for each use.

\subsection{Methods of characterization of the samples: $A$ - X-ray diffraction.}

The crystallite phase of the coated ferrofluid nanoparticles was identified by using X-ray diffraction patterns (XRD) using a PW 1800 Philips X-ray diffractometer equipped with a CuKa radiation $(\lambda=1.54056$ $\mathrm{nm})$.

\section{B- Transmission electron microscopy (TEM)}

The morphology of nanoparticles was examined using JEM -2100 electronic transmission microscope (TEM), operating at $200 \mathrm{kV}$ (accelerating voltage) and $1.43 \mathrm{~nm}$ (resolution wavelength). The samples were diluted in de- ionized water, then deposited on a carbon-coated copper TEM grid, and samples were left to airdry before TEM viewing.

\section{C- FT-IR Spectra.}

FT-IR Spectra was measured for all the samples(A,B,C,D and E) with a Fourier Transform Infrared Spectrometer (FT/IR -460 plus) in the wave number range $\left(4000-400 \mathrm{~cm}^{-1}\right)$.

\subsection{Dielectric properties}

The dielectric properties of all the samples were measured in the frequency range (from $0.5 \mathrm{kHz}$ to 1 $\mathrm{MHz}$ ) using a programmable automatic multi frequency RCL bridge (model PM6306), together with a cell type PW 9510/60, manufactured by Philips. The cell consists of two squared platinum black electrodes each having an area (A) of $0.8 \times 0.8 \mathrm{~cm}^{2}$ with an inter electrode distance (d) of $1 \mathrm{~cm}$.

The measured sample capacitance $\mathrm{C}$, resistance $\mathrm{R}$ and frequency $\mathrm{F}$ were used to calculate the dielectric constant $\left(\varepsilon^{\prime}\right)$,dielectric loss $\left(\varepsilon^{\prime \prime}\right)$, and conductivity $(\sigma)$.

It is important to know that the real part or dielectric constant $(\varepsilon)$ is mainly associated with the amount of polarization occurring in the material and the imaginary part $(\varepsilon)$ is a measure of dissipated energy. The dielectric performance of the material depends on the ionic, electronic, orientation and space charge polarization. The contribution to the space charge polarization appears due to the heterogeneity of the material ( Avanish Pratap Singh et al., 2011 ). 
The values of $\varepsilon^{\prime}, \varepsilon^{\prime \prime}$ and the ac conductivity $(\sigma) \mathrm{s}^{-1}$ were calculated through using the following equations (Polk and Postow 1995).

$$
\varepsilon^{\prime}=\frac{C d}{\varepsilon_{0} A}
$$

Where $\varepsilon_{0}$ is the permittivity of free space.

The dielectric loss $\left(\varepsilon^{\prime \prime}\right)$ of each sample was calculated as following .

$$
\varepsilon^{\prime \prime}=\varepsilon^{\prime} \tan \delta
$$

where Loss tangent $\tan \delta=1 / 2 \pi \mathrm{fCR}$

$$
\varepsilon^{\prime \prime}=\frac{\varepsilon}{2 \pi R F C}
$$

Moreover, the ac conductivity $\mathrm{S}\left(\mathrm{s}^{-1}\right)$ was calculated from the equation

$$
\sigma_{\mathrm{ac}}=\omega \varepsilon^{\prime \prime} \varepsilon_{\mathrm{o}}
$$

where $\omega=2 \pi \mathrm{f}$ is the angular frequency.

$\tau$ being the relaxation time, namely, the time at which the dielectric molecule has the ability to relax under the effect of the applied field and calculated from the relation

$$
\tau=\frac{1}{2 \pi \mathrm{f}_{\mathrm{c}}}
$$

$\mathrm{f}_{\mathrm{c}}$ being the critical frequency corresponding to the mid-point of the dispersion curve ( or the frequency at the maximum loss)(Fadel et al. 2003) . The accuracy of the experimental set-up was about $1 \rightarrow 3 \%$ in the whole frequency range investigated.

The difference between the value $\varepsilon^{\prime}{ }_{\text {s }}$ and $\varepsilon^{\prime}{ }_{\infty}$ at low and high frequency is called "The dielectric increment $\Delta \varepsilon^{\prime}$ ' i.e. This quantity is a measure for the shape and volume of the molecule ( Grant 1983 )

\subsection{Absorption spectra measurements:}

On the other hands, UV- visible spectrophotometer JASCO (V-550) was used for the measurements of absorption spectra in a range of wavelengths from (300-900) $\mathrm{nm}$ for all the prepared samples.

\section{Results and discussion}

The XRD patterns of $\mathrm{Fe}_{3} \mathrm{O}_{4}$ as in Figure (1) shows the characteristic diffraction peaks of $\mathrm{Fe}_{3} \mathrm{O}_{4}$. The diffraction peaks are broadened owing to very small crystallite size ( H. Kavas et al., 2010 ). The peaks observed at $2 \theta=30.027^{\circ}, 35.581^{\circ}, 43.251^{\circ}, 53.519^{\circ}, 57.175^{\circ}$ and $62.698^{\circ}$ are assigned to pure $\mathrm{Fe}_{3} \mathrm{O}_{4}$ according to literature (Jianguo Deng et al., 2003).The grain size D of the $\mathrm{Fe}_{3} \mathrm{O}_{4}$ particles was calculated by Scherrer's equation: $\mathrm{D}=(0.89 \lambda) /(\beta \cos \theta)$, where $\lambda$ is the $\mathrm{X}$-ray wavelength $\left(\lambda=1.5406^{\circ} \mathrm{A}\right), \beta$ is the full-width at half maxima (FWHM) of the X-ray diffraction peak, $\theta$ is Bragg angle in degrees ( Javed Alam et al., 2007 ) .The particle size was calculated to be about $10.375 \mathrm{~nm}$, which confirms the nanosize of ferrite.

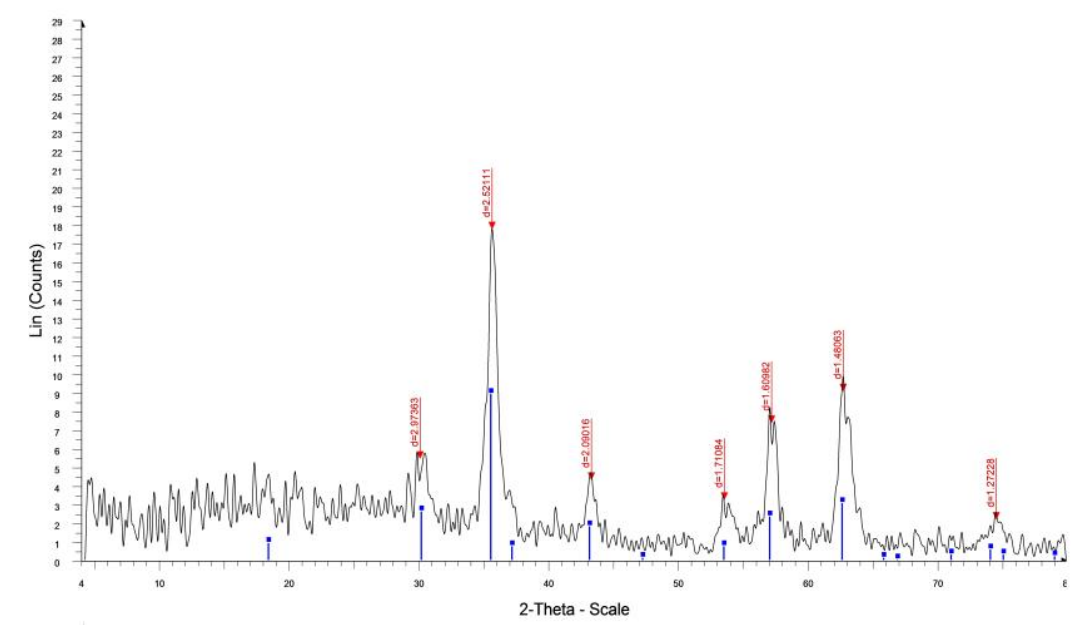

Fig. (1): XRD patterns of the PEG-coated magnetite nanoparticles 
Using of Transmission electron microscope (TEM) technique to explain the aggregation and the shape of conjugation of Dox. with ferrofluid. Fig (2.a) show the shape of the nanomagnetic particle ferrofluid alone and Fig (2. B,C,D and E)) shows the shape of the conjugation samples (B,C,D and E) respectively. The results explain that ferrofluid nanoparticles spread within Dox molecules and conjugated with it and the darkening in the figure is due to increasing the amount of ferrofluid magnetic nanoparticles.

On the other hand, FT-IR spectroscopy has long been recognized as a powerful tool for elucidation of structural information. The position, intensity and shape of vibration bands are useful in clarifying conformational and environmental changes of the studied material at the molecular level ( Ki Lee et al., 1994 ). Herein, the samples of ferrofluid, $\mathrm{Fe}_{3} \mathrm{O}_{4}$, and doxorubicin have been investigated by FTIR spectroscopy which its spectra are shown in Figure (3).
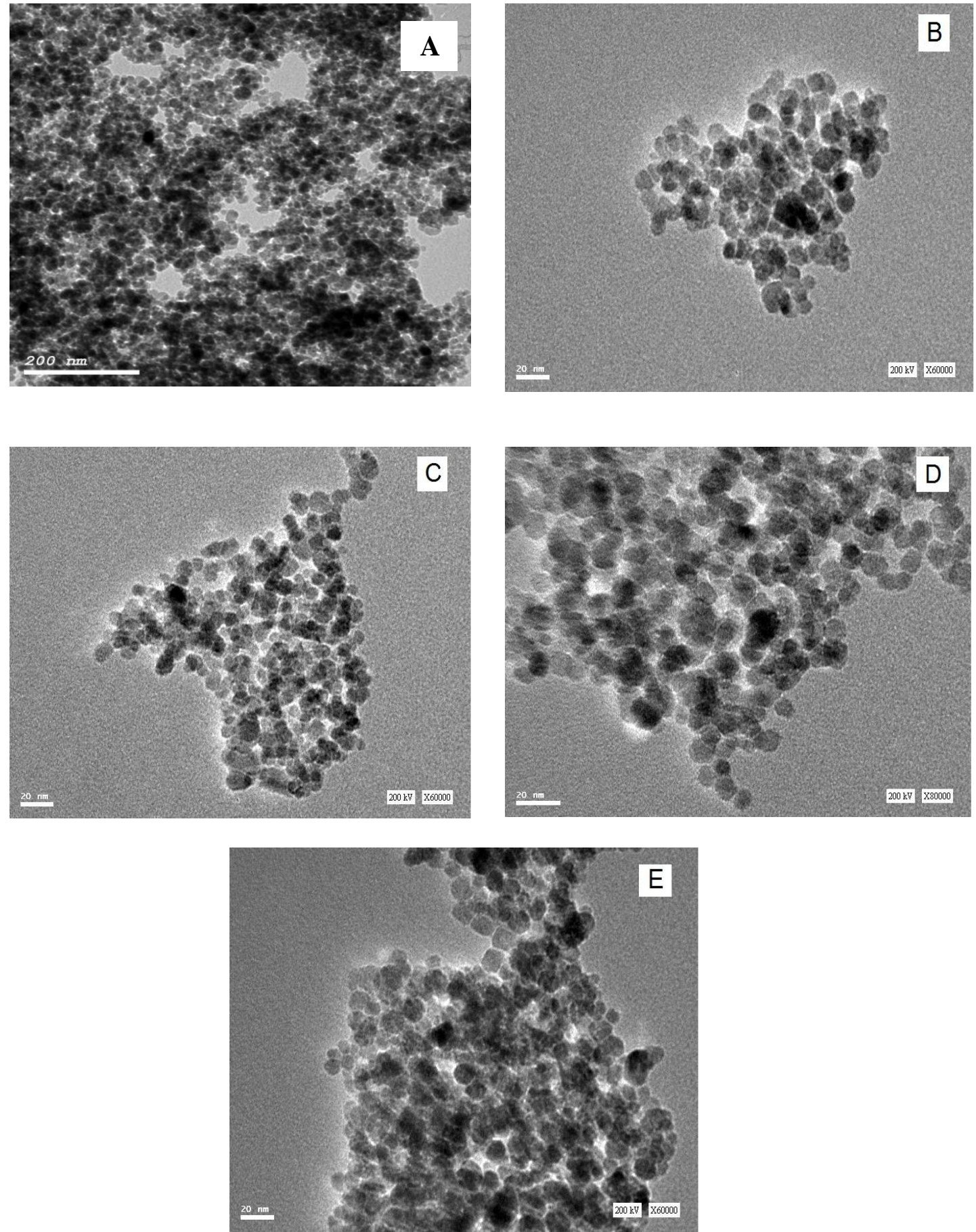

Fig. (2): TEM images of (a) ferrofluid, (b-e) different amounts of ferrofluid loaded by doxorubicin. 
The curves illustrate the characteristic peaks that are exhibited by the ferrofluid in which the metal-oxygen band, observed at $590 \mathrm{~cm}^{-1}$ corresponds to intrinsic stretching vibrations of the metal ions at tetrahedral site $\left(\mathrm{Fe}_{\text {tetra }} \leftrightarrow \mathrm{O}\right)$, whereas metal-oxygen band observed at $436.1 \mathrm{~cm}^{-1}$, is assigned to octahedral metal- ion stretching $\left(\mathrm{Fe}_{\text {octa }} \leftrightarrow \mathrm{O}\right)$ in agreement with literature ( Kavas et al., 2010 ).

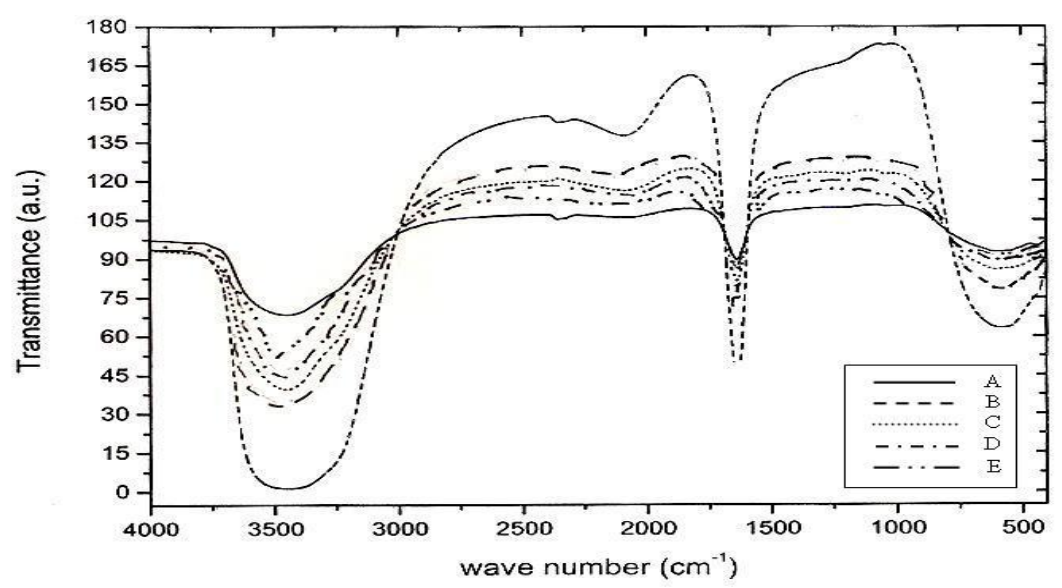

Fig. (3): FTIR spectrum of samples A,B,C,D and E

Moreover, the band at $1637 \mathrm{~cm}^{-1}$ is assigned to bending $(\delta)$ vibrations due to adsorbed water on the surface of the iron oxide nano-particles. Also, $\mathrm{OH}$ stretching/vibration band observed at around $3450 \mathrm{~cm}^{-1}$ has verified the physical adsorption of PEG chains onto the $\mathrm{Fe}_{3} \mathrm{O}_{4}$ nanoparticle surface in accordance with the literature ( Feng B et al., 2008 ).

Alternatively, FTIR spectrum of pure doxorubicin shows peak at $3459 \mathrm{~cm}^{-1}$ due to $\mathrm{N}-\mathrm{H}$ stretching vibrations for the primary amine structure. Also, the band at $1636 \mathrm{~cm}^{-1}$ is assigned to $\delta(\mathrm{N}-\mathrm{H})$ (Kayal et al., 2010 ). On the other hand, FT-IR spectra of doxorubicin loaded on the ferrofluid shows an insignificant shifting in the positions of the peaks of ferrofluid and also of the doxorubicin as well as a change in the intensity of the their peaks which indicates an interaction between them.

The dielectric properties of the samples are shown in Figures (4) which illustrate the variation of $\varepsilon^{\prime}, \varepsilon^{\prime \prime}$ and $(\sigma)$ as a function of applied frequency for all the samples A, B, C, D and E, respectively. The results indicated that all the samples measured have a dielectric dispersion in the applied frequency range from $0.5 \mathrm{kHz} \rightarrow 1$ $\mathrm{MHz}$ a phenomenon previously measured by Grant (1983). It is clear that the values of $\varepsilon$ ' were sharply decreased with frequency while remained nearly unchanged at higher frequencies (beyond $1 \mathrm{kHz}$ ). The dielectric permittivity in the lower range of frequencies was observed to be strongly dependent on frequency as well as the ferrofluid content. In other words, the higher values of $\varepsilon$ ' were observed at low frequencies because of the low resistivity of magnetite, which generated electric charge transference ( Ramajo et al., 2009) 

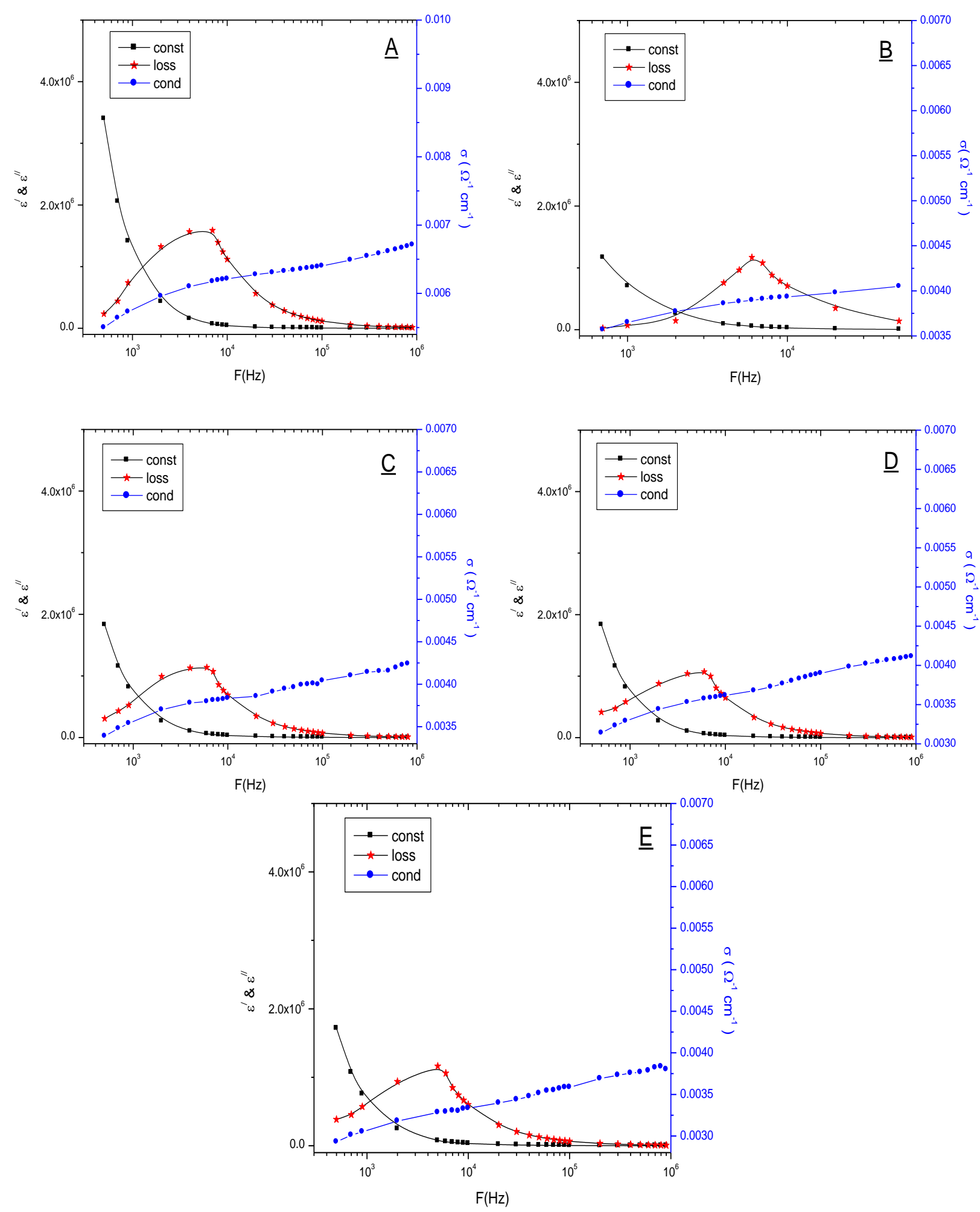

Fig. (4): The variation of the dielectric constant, $\varepsilon^{\prime}$, dielectric loss, $\varepsilon^{\prime \prime}$, and ac conductivity, $\sigma$ for all studied samples.

Dielectric properties are mainly governed by the conduction mechanism in ferrites, wherein electron hopping takes place. This electron hopping looks to be favorable at lower applied electric field frequencies. Therefore, at lower frequency the dielectric constant is maximum. However, at higher frequency hopping electrons could not 
follow the rate of alternation of applied field and hence show dispersion with increase in applied frequency ( Lipare et al., 2003 ).

In other words, the polarization in ferrites is inferred from electron exchange between $\mathrm{Fe}^{2+}$ and $\mathrm{Fe}^{3+}$, the local displacement of electrons in the direction of the applied field takes place and these electrons establish the polarization. By increasing the frequency, the polarization decreases and reaches a fairly stable value because the electron exchange between $\mathrm{Fe}^{2+}$ and $\mathrm{Fe}^{3+}$ cannot go after the alternating field. Indeed, the decrease in dielectric constant with frequency is expected as a consequence of the fact that any species contributing to polarizability is found to show lagging behind the applied field at higher and higher frequencies ( Kavas et al., 2010 ).

Ac conductivity versus frequency for various concentrations of doxorubicin- ferrofluid samples (B, C, $\mathrm{D}$ and $\mathrm{E}$ ) and pristine doxorubicin sample (A) comprise conductivity plateau regions at room temperature which illustrate a strongly dependent of the conductivity values on applied frequency below $1 \mathrm{kHz}$ while a weak dependent was observed above $1 \mathrm{kHz}$.

Furthermore, a clear decrease in the values of the conductivity was observed by increasing the ferrite contents which can be attributed to the insulating behavior of the iron oxide in the core of the nanoparticles, which hinders the charge transfer thereby lowering the conductivity ( Javed Alam et al., 2007 ). Besides, when $\mathrm{Fe}_{3} \mathrm{O}_{4}$ nanoparticles contents increases the interactions between the doxorubicin and iron oxide nanoparticles increase the charge carrier scattering and thus increase the sample resistivity which could also splay a role in the reduction of the conductivity ( Yunze Long et al., 2005 ).

Also, it is clear from the figures that the electric conductivity $(\sigma)$ for all the samples increased with the increase of the frequency, all figures show the conductivity dispersion due to the interfacial polarization (Gabriel (1996). The change in the critical frequency $f_{c}$ (consequently in the relaxation time $\tau$ ) shown in all the figures is attributed to the change in the average molecular radii. From the data in the figures and through the use of the equations 1,3 and 4 for all the samples, the relaxation time $\tau$, the dielectric increment, were calculated as given in Table(1). The changes in the values of the dielectric increment $\Delta \dot{\varepsilon}$ for all the samples indicates changes in both shape and volume of the molecules (Grant 1983).

\begin{tabular}{|c|c|c|c||c||}
\hline Sampleq & $\mathrm{F}_{\mathrm{c}}(\mathrm{Hz})$ & $\tau(\mathrm{sec})$ & $\boldsymbol{\sigma}$ & $\Delta \boldsymbol{\varepsilon}^{\prime}$ \\
\hline \hline $\mathrm{A}$ & 7000 & $2.27479 \times 10^{-5}$ & 0.00671 & $3.400714968 \times 10^{6}$ \\
\hline \hline $\mathrm{B}$ & 6800 & $2.34170 \times 10^{-5}$ & 0.00425 & $1.1644 \times 10^{6}$ \\
\hline \hline $\mathrm{C}$ & 6500 & $2.4497 \times 10^{-5}$ & 0.00424 & $1.82901488 \times 10^{6}$ \\
\hline \hline $\mathrm{D}$ & 6000 & $2.6539 \times 10^{-5}$ & 0.00411 & $1.837884792 \times 10^{6}$ \\
\hline $\mathrm{E}$ & 5000 & $3.18471 \times 10^{-5}$ & 0.00383 & $1.716405807 \times 10^{6}$ \\
\hline
\end{tabular}

Table (1):The dielectric increment $\Delta \varepsilon^{\prime}$ the relaxation time $\tau$ and conductivity $\boldsymbol{\sigma}$ for all studied samples.

The variation of the dielectric loss for all the samples is studied as a function of applied frequency and the results explained that a decrease was observed in the values of dielectric loss of doxorubicin by increasing the ferrofluid content loaded with it. In fact, the dielectric losses in ferrites are generally reflected in the resistivity measurement. Materials with high resistivity exhibit low dielectric losses and vice versa.

Therefore, the observed decrease of loss tangent values by increasing ferrofluid content confirms the increase of resistivity supporting the Verway conduction mechanism( Lipare 2003) .

Interestingly, it can be seen from fig (2) that there is a strong correlation between the conduction mechanism and the dielectric behavior of ferrites. The conduction mechanism in ferrites is considered as due to hopping of electrons between $\mathrm{Fe}^{2+}$ and $\mathrm{Fe}^{3+}$. When the hopping frequency is nearly equal to that of the frequency of externally applied electric field, a maximum of dielectric loss may be observed. The condition for observing a maximum in the dielectric losses of a dielectric material is given by $\omega \tau=1$, where $\omega$ is the $2 \pi f_{c}$ and $\tau$ is the relaxation time. The relaxation time is related to the jumping probability per unit time, $\mathrm{P}$, by the equation $\tau=1 / 2 \mathrm{P}$ or $\mathrm{f}_{\max } \alpha \mathrm{P}$. This shows that $\mathrm{f}_{\max }$ is proportional to the jumping probability or hopping probability (Iwauchi 1971 and Humaira et al 2010)

Figure (5) shows the absorption spectra for the studied samples measured in a range of wavelengths from 300 to $900 \mathrm{~nm}$. A peak was observed at wavelength $481.1 \mathrm{~nm}$ for the pristine sample A in agreement with the ( JAYITA BHATTACHARJEE et al., 2008 ) which was shifted towards low wavelength by loading the doxorubicin by different content of ferrofluid. 


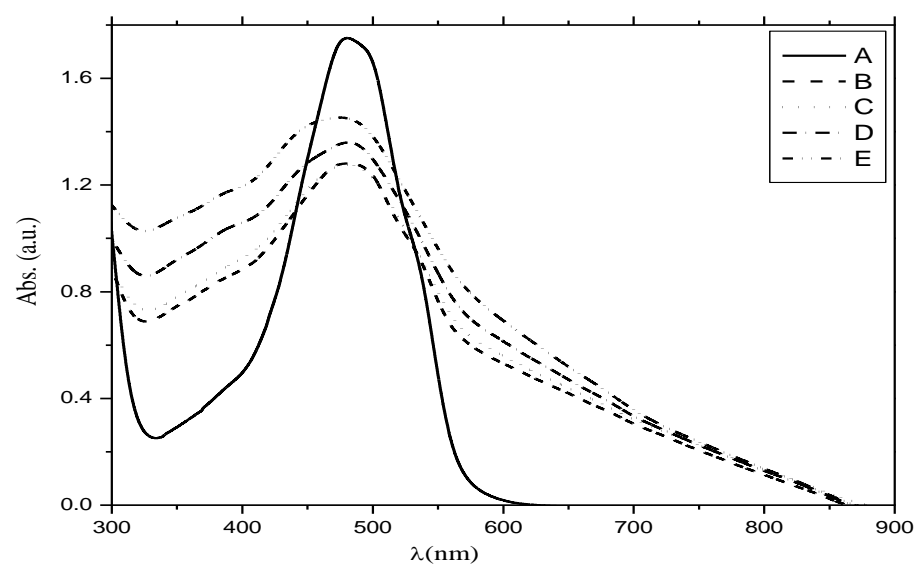

Fig. (5): Absorption spectra of different amounts of ferrofluid loaded by doxorubicin at room temperature.

It could be observed that the absorption peak increased by increasing the ferrofluid content loaded with the doxorubicin. In addition, the full width at half maximum ( $F W H M$ ) was calculated and listed in Table (2). It can be clearly shown that the absorption bandwidth became broader by increasing the ferrofluid content. This may be due to the inhomogeneous distribution of the ferrofluid in the doxirubicin. The distribution of ferrofluid nanoparticle surrounded by doxorubicin media have subtly different chemical and dielectric character which causes this inhomogeneous broadening ( Ahmed et al., 2013 ).

Table (2): Optical properties of different amounts of ferrofluid loaded by doxorubicin.

\begin{tabular}{|c|c|c|c|}
\hline Sample & $\begin{array}{c}\lambda_{\max } \\
(\mathrm{nm})\end{array}$ & $\begin{array}{c}\text { Abs. } \\
(\text { a.u. }\end{array}$ & $\begin{array}{c}\text { FWHM } \\
(\mathrm{nm})\end{array}$ \\
\hline $\mathrm{A}$ & 481.1 & 1.76 & 94.6 \\
\hline $\mathrm{B}$ & 480.6 & 1.28 & 103.2 \\
\hline $\mathrm{C}$ & 480.0 & 1.28 & 104.9 \\
\hline $\mathrm{D}$ & 479.5 & 1.36 & 106.6 \\
\hline $\mathrm{E}$ & 478.0 & 1.45 & 108.4 \\
\hline
\end{tabular}

\section{Conclusion}

We can conclude that their is no great changes in the properties of the different samples. Then conjugation of DOX with any amounts of ferrofluid can be used in medical applications.

\section{References}

[1]. Ahmed R. M. and M. Saif (2013) Optical Properties of Rhodamine B Dye Doped in Transparent Polymers for Sensor Application,CHINESE JOURNAL OF PHYSICS, VOL. 51, NO. 3, 511-521.

[2]. Avanish Pratap Singh, Monika Mishra, Amita Chandra and S K Dhawan(2011) Graphene oxide/ferrofluid/cement composites for electromagnetic interference shielding application, Nanotechnology, 22,465701 (9pp)

[3]. Fadel M A, Wael S M , Mostafa (2003) Effect of $50 \mathrm{~Hz}, 0.2 \mathrm{mT}$ Magnetic field Fields on RBC properties and Heart Functions of Albino Rats. Bioelecomagnetics, 24, 535-545.

[4]. Feng B, Hong RY, Wang LS, Guo L, Li HZ, Ding J, Zheng Y, Wei DG (2008) Synthesis of Fe3O4/APTES/PEG diacid functionalized magnetic nanoparticles for MR imaging, Colloids and Surfaces A: Physicochemical and Engineering Aspects, 328, $52-59$.

[5]. Gabriel C., Gabriel S. and Corthout E. (1996) The dielectric properties of biological tissue, I-Literature survey Phys. Med. Biol., 41: 2231-49.

[6]. Grant E.H. (1983 ) Molecular interpretation of the dielectic behavior of biological material, Biological Effect and Dosimetry of Non-ionizing Radiation New York and London. Pelnum.

[7]. Harris J.M., Chess, R.B. (2003) Effect of pegylation on pharmaceuticals, Nat. Rev. Drug Discov, 2, 214-221.

[8]. Herve K., L. Douziech-Eyrolles, E. Munnier, S. Cohen-Jonathan, M. Souce, H. Marchais, P. Limelette, F. Warmont, M. Saboungi, P. Dubois, I. Chourpa (2008) The development of stable aqueous suspensions of PEGylated SPIONs for biomedical applications, Nanotechnology $19,465608.465607 \mathrm{pp}$.

[9]. Humaira Anwar, Asghari Maqsood (2010) TEMPERATURE DEPENDENT STRUCTURAL AND ELECTRICAL ANALYSIS OF Mn-Zn NANO FERRITES ,J Pak Mater Soc, 4, 2,p81-94.

[10]. Iwauchi K Jap.(1971) Dielectric Properties of Fine Particles of $\mathrm{Fe}_{3} \mathrm{O}_{4}$ and Some Ferrites,J .Appli. Phys. ,10, 1520-1528.

[11]. Javed Alam, Ufana Riaz, Sharif Ahmad (2007) Effect of ferrofluid concentration on electrical and magnetic properties of the Fe3O4/PANI nanocomposites, Journal of Magnetism and Magnetic Materials, 314 , 93-99.

[12]. Jayita Bhattacharjee, Gunjan Verma, V K Aswal and P A Hassan (2008) Small angle neutron scattering study of doxorubicinsurfactant complexes encapsulated in block copolymer micelles, PRAMANA - journal of physics ,Vol.71,pp.991-995. 
[13]. Jianguo Deng, ChuanLan He, Yuxing Peng, Jianhua Wang, Xingping Long, Pei Li, Albert S.C. Chan (2003) Magnetic and conductive Fe3O4-polyaniline nanoparticles with core-shell structure, Synthetic Metals, 139, 295-301.

[14]. Karakoti A.S., S. Das, S. Thevuthasan, S. Seal (2011) PEGylated inorganic nanoparticles, Angew. Chem. Int. Ed. Engl,50 , 19801994.

[15]. Kavas H., Z. Durmus, A. Baykal, A. Aslan, A. Bozkurt, M.S. Toprak (2010) Synthesis and conductivity evaluation of $\mathrm{PVTri}^{-} \mathrm{Fe}_{3} \mathrm{O}_{4}$ nanocomposite,Journal of Non-Crystalline Solids, 356, 484-489.

[16]. Kayal S., R.V. Ramanujan (2010) Doxorubicin loaded PVA coated iron oxide nanoparticles for targeted drug delivery,Materials Science and Engineering C, $30,484-490$.

[17]. Ki-Lee W. and C. S. Ha (1994) Korea Polymer Journal, vol. 8, no. 6, pp. 934-941.

[18]. Lankveld D.P., R.G. Rayavarapu, P. Krystek, A.G. Oomen, H.W. Verharen, T.G. van Leeuwen, W.H. De Jong, S. Manohar (2011) Blood clearance and tissue distribution of PEGylated and non-PEGylated gold nanorods after intravenous administration in rats, Nanomedicine (Lond), $6,339-349$.

[19]. Lipare A.Y., P.N. Vasambekar, A.S. Vaingankar (2003) Dielectric behavior and a.c. resistivity study of humidity sensing ferrites , Materials Chemistry and Physics $81,108-115$.

[20]. Polk C \& Postow E (1995) Hand book of biological effects of electromagnetic fields, 2nd edn, pp. 99-107, CRC Press Boca Raton , New York.

[21]. Ramajo L.A., A.A. Cristóbal, P.M. Botta, J.M. Porto López, M.M. Reboredo, M.S. Castro (2009) Dielectric and magnetic response of $\mathrm{Fe}_{3} \mathrm{O}_{4}$ /epoxy composites, Composites: Part A: Applied Science and Manufacturing , 40, 388-393.

[22]. Rosensweig R. E.(2002) Heating magnetic fluid with alternating magnetic field, Journal of Magnetism and Magnetic Materials, Vol.252, 307-374.

[23]. Shan X., Y. Yuan, C. Liu, X. Tao, Y. Sheng, F. Xu (2009) Influence of PEG chain on the complement activation suppression and longevity in vivo prolongation of the PCL biomedical nanoparticles, Biomed. Microdev., 11, 1187-1194.

[24]. Wang, M., Thanou, M., (2010) Targeting nanoparticles to cancer,Pharmacol. Res. 62, 90-99.

[25]. Yunze Long, Zhaojia Chen, Jean Luc Duvail, Zhiming Zhang, Meixiang Wan(2005) Electrical and magnetic properties of polyaniline/ $\mathrm{Fe}_{3} \mathrm{O}_{4}$ nanostructures, Physica B : Condensed Matter, 370,121-130. 